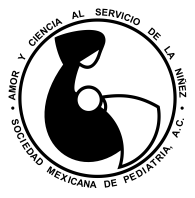

\title{
Linfangioma cervicofacial, detección prenatal
}

\author{
Mirna Alicia Salas-Samaniego, ${ }^{\ddagger} *$ Manuel Cázarez-Ortiz, ${ }^{\S}$ María Adela Ramírez-Moreno॥ \\ ‡ Residente de Neonatología; § Profesor titular del curso de Neonatología; \\ "l Neonatóloga. Centro Médico Nacional 20 de Noviembre, ISSSTE. Ciudad de México, México.
}

\begin{abstract}
RESUMEN
Objetivo: Describir el caso de un paciente con linfangioma cervicofacial detectado durante el embarazo y que recibió tratamiento con OK-432 poco después del nacimiento. Presentación del caso: Neonato con detección de defecto anatómico cervical por ultrasonido a las 26 semanas de gestación; mediante otros estudios de imagen como resonancia magnética se sospechó linfangioma. El embarazo llegó a término, el cual se resolvió por cesárea sin complicaciones. Al nacimiento se confirmó el diagnóstico de linfangioma cervicofacial micro y macroquístico, por lo que a los ocho días de vida se aplicó escleroterapia con OK-432. La lesión se redujo significativamente después de cinco y 10 semanas de tratamiento. El paciente evolucionó de manera satisfactoria, sin complicaciones. Conclusiones: Dado que los linfangiomas pueden ser detectados en el periodo fetal, los pacientes pueden recibir tratamiento con OK-432 desde la etapa neonatal temprana.
\end{abstract}

Palabras clave: Linfangioma, OK-432, esclerosis, diagnóstico prenatal, recién nacido.

\section{INTRODUCCIÓN}

Las malformaciones del sistema linfático ocurren de 2.8 a 5 de cada 100,000 recién nacidos vivos y corresponden a 5.6\% de todas las lesiones benignas de la infancia a nivel mundial. Las más frecuentes son los linfangiomas, y se localizan hasta en $75 \%$ en la cabeza y cuello, otras áreas descritas son lengua y orofaringe; sin diferencia

\footnotetext{
* Correspondencia: MASS, mirna.salas.s@gmail.com Conflicto de intereses: Los autores declaran que no tienen. Citar como: Salas-Samaniego MA, Cázarez-Ortiz M, RamírezMoreno MA. Linfangioma cervicofacial, detección prenatal. Rev Mex Pediatr. 2020; 87(1):26-29. doi: 10.35366/93265 [Facial-cervical lymphangioma, prenatal detection]
}

\begin{abstract}
Objective: To describe the case of a newborn with cervicalfacial lymphangioma detected during pregnancy and who received treatment with OK-432 shortly after birth. Case presentation: Newborn with detection of cervical anatomic defect by ultrasound at 26 weeks of gestation; lymphangioma was suspected using other studies such as magnetic resonance imaging. The pregnancy came to term, which was stirred by cesarean section without complications. At birth and after performing other studies, the diagnosis of micro and macrocystic cervical-facial lymphangioma was confirmed. At eight days of age sclerotherapy with OK-432 was applied. After five and 10 weeks of treatment the lesion was significantly reduced. The patient evolved satisfactorily, without complications. Conclusions: Since lymphangiomas can be detected in the fetal period, in the early neonatal stage patients can receive treatment with OK-432.
\end{abstract}

Keywords: Lymphangioma, OK-432, sclerosis, prenatal diagnosis, newborn.

en cuanto al sexo. ${ }^{1-3}$ En el Centro Médico Nacional 20 de Noviembre se han registrado dos pacientes con detección prenatal de este tipo de malformaciones en los últimos cinco años.

Aproximadamente en el $50 \%$ de los casos, esta condición es evidente al nacimiento y hasta en $90 \%$ están presentes a los dos años de edad. Los linfangiomas se relacionan con malformaciones cardiacas, renales y deformidades esqueléticas, pero además se presentan con frecuencia en pacientes con trisomía 13, 18 y 21, así como en síndrome de Turner o Noonan. ${ }^{1,4,5}$

Investigaciones recientes han descrito que el origen de estas anomalías son mutaciones somáticas, principalmente en el gen PIK3CA, que se asocia 
con sobrecimiento tisular, células de mayor tamaño y algunos tumores malignos. ${ }^{6}$ Se describen cuatro tipos histológicos: 1) higroma quístico o linfangioma macroquístico, compuesto por grandes espacios linfáticos macroscópicos con revestimiento de colágeno y músculo liso; 2) cavernoso o microquístico, constituido por pequeños vasos linfáticos con capas conectivas de grosor irregular; 3) capilar o simple, formado por vasos linfáticos de paredes delgadas y; 4) malformación vasculolinfática, de componente linfático y de componente vascular. ${ }^{2,7}$

Algunos linfangiomas tienden a invadir estructuras anatómicas contiguas, por lo que la tomografía y resonancia magnética (IRM) son útiles en la evalua-

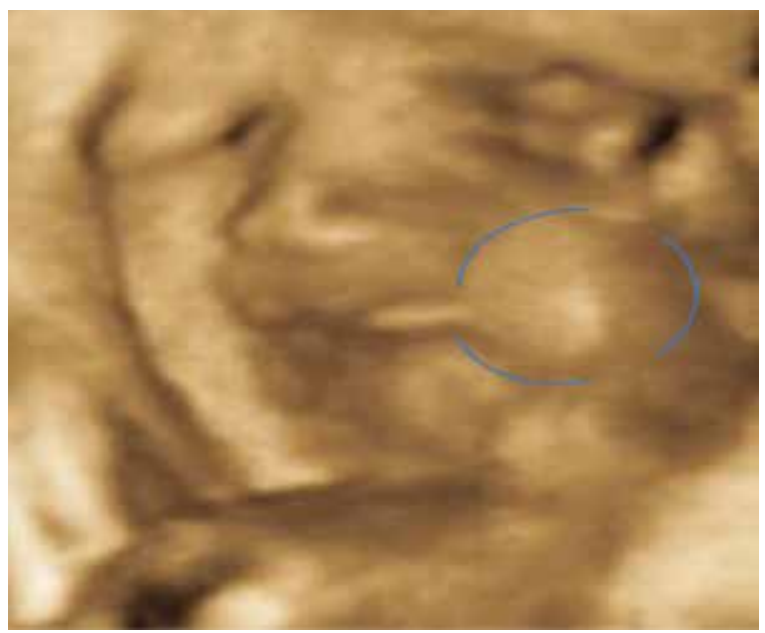

Figura 1: Ultrasonido prenatal 3D donde se observa masa en región cervicofacial del lado izquierdo.

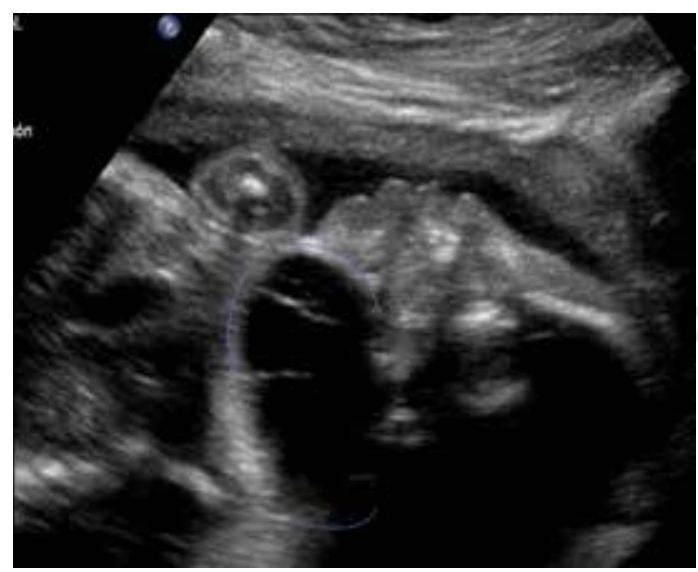

Figura 2: Ultrasonido obstétrico.

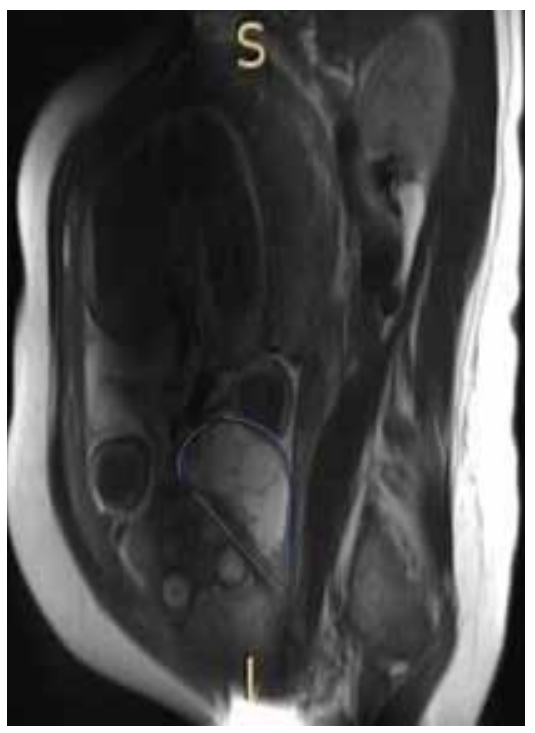

Figura 3: Resonancia magnética con linfangioma quístico cervicofacial.

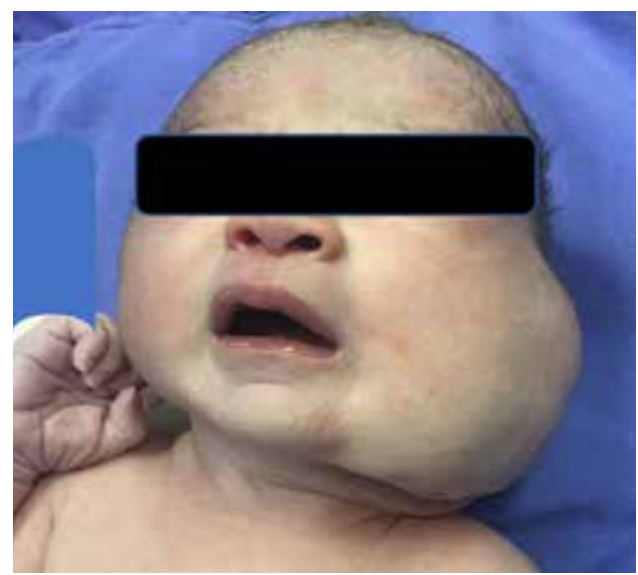

Figura 4: Recién nacido con masa en región cervicofacial izquierda.

ción de la extensión y profundidad, principalmente para evaluar estructuras críticas, como la vía aérea, así como para guiar decisiones terapéuticas. ${ }^{1,2,5}$ En la década de 1990 se recomendaba la resección quirúrgica como tratamiento de elección; sin embargo, este manejo puede ocasionar lesiones nerviosas o vasculares, y tiene una elevada tasa de recurrencia. Por esta razón, la infiltración de sustancias esclerosantes con tetradecilsulfato sódico, etanol, bleomicina, o bien con OK-432, ha demostrado ser una alternativa terapéutica con resultados favorables. ${ }^{8}$ 

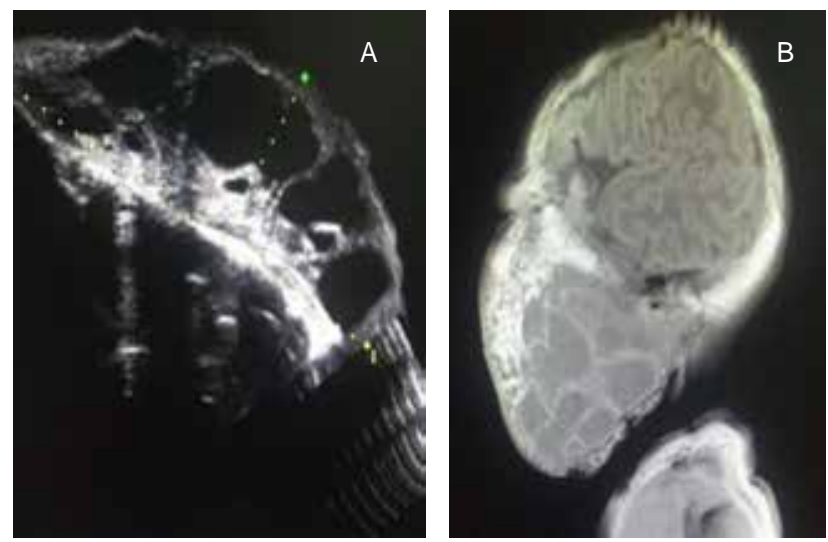

Figura 5: A) Ultrasonido postnatal. B) Resonancia magnética con linfangioma, sin compresión de vía aérea.

\section{PRESENTACIÓN DEL CASO}

Reportamos el caso de un neonato, producto de madre primigesta de 29 años y padre de 34 , ambos en buen estado de salud y sin antecedentes patológicos relevantes, quien fue detectado a las 26 semanas de gestación (SDG) con un defecto anatómico cervical, que presuntivamente era encefalocele, por lo que la madre fue referida a un hospital de tercer nivel de atención.

En el servicio de Medicina Materno Fetal a las 28 SDG se realizaron ultrasonidos seriados (Figuras 1 y 2), en los cuales se identificaron imágenes compatibles con linfangioma cervical de 89 × 61 x $36 \mathrm{~mm}$, de componente mixto, sin compromiso aparente de vía aérea. Posteriormente, a las 35 SDG por IRM se reportó linfangioma quístico cervicofacial, de 6.5 x 5.7 x $5.9 \mathrm{~cm}$, sin que se detectara compromiso de la vía aérea (Figura 3). Por la posibilidad de componente genético se realizó cariotipo prenatal, el cual resultó como 46, XY.

El nacimiento del paciente ocurrió a término mediante una cesárea programada. Se recibió producto único, que lloró y respiró espontáneamente, con peso de 3,400 gramos y talla de $51 \mathrm{~cm}$. A la exploración física se observó un recién nacido normocéfalo, sin alteraciones oculares y con adecuada implantación de pabellones auriculares. Por supuesto, se detectó asimetría cervicofacial izquierda secundaria a tumoración que estaba localizada desde el arco cigomático hasta la región submandibular, de consistencia blanda, desplazable, no dolorosa y cubierta por piel normal, con medidas de $9 \times 7 \mathrm{~cm}$ (Figura 4). No se observaron malformaciones en tórax, abdomen, genitales ni extremidades. En su primera hora de vida fue valorado por otorrinolaringología pediátrica, se exploró la cavidad oral y la vía aérea por laringoscopía, descartando compromiso alguno.

Se realizó ultrasonido e IRM después del nacimiento (Figuras $5 A$ y 5B), corroborando diagnóstico de linfangioma cervicofacial izquierdo, de componente mixto, con presencia de múltiples microquistes y una lesión macroquística, con dimensiones de 56 × 33 × $60 \mathrm{~mm}$ en sentido longitudinal, transversal y anteroposterior. El reporte de ecocardiograma postnatal descartó defectos septales.

El servicio de Cirugía Pediátrica determinó que por las características de la lesión, la opción sería la aplicación de escleroterapia con OK-432 (picibanil). A los ocho días de vida, previa aplicación de anestesia local, se puncionó la lesión (guiada por transiluminación), extrayendo $15 \mathrm{~mL}$ de líquido xantocrómico, con posterior administración de $20 \mathrm{~mL}$ de solución de picibanil, sin complicaciones durante el procedimiento.

El paciente presentó en las primeras 24 horas inflamación y eritema de la lesión, acompañadas de

Figura 6:

Paciente con reducción evidente del tamaño del linfangioma.
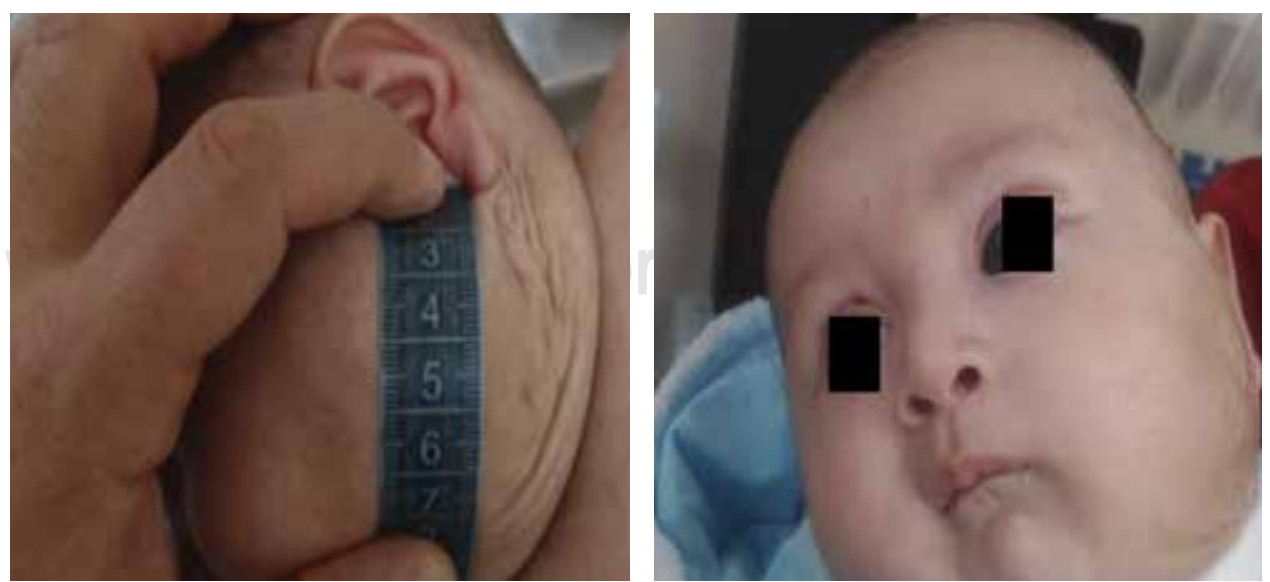
hipertermia de $37.8{ }^{\circ} \mathrm{C}$, manejadas con paracetamol, con mejoría paulatina. Se decidió su egreso hospitalario 72 horas después. A las cinco semanas del tratamiento con OK-432, la lesión se redujo de 9 × 7 a 7 x 6 cm y, a las 10 semanas sus medidas fueron $6 \times 4.5 \mathrm{~cm}$ (Figura 6). A los dos meses y 16 días de edad, el paciente continúa en seguimiento sin presentar signos de recidiva del linfangioma, llevando a cabo una vida normal para su edad, y sin problemas para su alimentación.

\section{DISCUSIÓN}

El tratamiento clásico del linfangioma consiste en la escisión quirúrgica, con el objetivo de preservar las estructuras vasculares y nerviosas involucradas; sin embargo, el daño a éstas puede ocurrir durante la cirugía o en el postoperatorio, como fístulas, infecciones o dehiscencia de la herida. Además, la mortalidad puede ser hasta de $6 \%$ y la recurrencia hasta de $27 \%{ }^{9}$ Las limitaciones del manejo quirúrgico han llevado al desarrollo de otros tratamientos, principalmente la escleroterapia, cuyo propósito es la regresión parcial o total del linfangioma. ${ }^{9}$ Aún no existen guías ni consensos establecidos para el tratamiento de estas malformaciones, por lo que depende de la experiencia del centro de atención, la decisión de los padres y la individualización de cada caso. ${ }^{10}$

El principio de la escleroterapia se basa en una reacción inflamatoria en el endotelio, que conduce a trombosis, obstrucción luminal, cicatrización y finalmente reducción del tamaño. Las sustancias aprobadas son el OK-432, la bleomicina y la doxiciclina. ${ }^{10,11} \mathrm{En}$ 1986, Ogita y colaboradores observaron la efectividad de OK-432 con reducción y resolución completa sin necesidad de cirugía. ${ }^{11}$ OK-432 se obtiene de Streptococcus pyogenes del grupo A; la dosis máxima a aplicar es de $0.2 \mathrm{mg}$ diluidos en $20 \mathrm{~mL}$ de solución fisiológica. Se sugieren intervalos de dos a 12 semanas entre cada inyección. ${ }^{3,11}$

En 2012, Rodríguez y su equipo analizaron a 15 pacientes entre 12 días y 12 años de edad, en $40 \%$ de ellos se encontró linfangioma macroquístico y $46.6 \%$ mixtos, manejados con OK-432. Se lograron resultados favorables con un máximo de tres dosis con excelente resultado en $33.4 \%$; en el resto el resultado no fue el esperado, lo que se asoció a que contaban con cirugía previa y tipo de linfangioma microquístico. ${ }^{8}$ Valle Rodríguez reportó el caso de un adolescente de 16 años con un linfagioma macroquístico de igual localización a la de nuestro paciente, con dimensiones de $10 \times 6 \mathrm{~cm}$, tratado con una dosis de OK-432; a las 16 semanas del tratamiento ya no se apreciaba clínicamente y en 42 semanas no se detectó recidiva. ${ }^{3}$ En general, no se recomienda más de tres dosis OK-432, ya que se ha demostrado que mayor número de dosis no resulta efectivo. ${ }^{11}$

Respecto a otros agentes esclerosantes, el más estudiado es la bleomicina. En 2008 se hizo un metaanálisis de 22 series de casos, que comparó el uso de OK-432 con la bleomicina, en el cual no se encontró diferencia estadísticamente significativa; sin embargo, con bleomicina puede haber efectos colaterales como fibrosis pulmonar, lo cual no se ha descrito con OK- $432 .^{3,9}$

\section{REFERENCIAS}

1. Waner M, O TM. Multidisciplinary approach to the management of lymphatic malformations of the head and neck. Otolaryngol Clin North Am. 2018; 51(1): 159-172.

2. Teissier N. Linfangiomas cervicofaciales: pruebas de imagen, diagnóstico y tratamiento. EMC Otorrinolaringología. 2017; 46: 1-15.

3. Valle-Rodríguez, E, Villanueva-San Vicente, V, RodríguezGonzález MA et al. Linfangioma cervical: manejo terapéutico con OK-432 (picibanil). Rev Esp Cir Oral y Maxilofac. 2007; 29: 399-403.

4. Tongsong T, Luewan S, Khorana J, Sirilert S, Charoenratana C. Natural course of fetal axillary lymphangioma based on prenatal ultrasound studies. J Ultrasound Med. 2018; 37(5): 1273-1281.

5. Bansal AG, Oudsema R, Masseaux JA, Rosenberg HK. US of pediatric superficial masses of the head and neck. Radiographics. 2018; 38(4): 1239-1263.

6. Perkins JA. New frontiers in our understanding of lymphatic malformations of the head and neck (Natural History, basic research). Otolaryngol Clin North Am. 2018; 51(1): 147-158.

7. Gimeno-Aranguez M, Colomar-Palmer P, González-mediero I et al. Aspectos clínicos y morfológicos de los linfangiomas infantiles: revisión de 145 casos. An Esp Pediatr. 1996; 45: 25-28.

8. Rodríguez J, Cáceres F, Vargas P. Manejo del linfangioma con infiltración de OK-432. Cir Pediatr. 2012; 25: 201-204.

9. De Oliveira-Olímpio H, Bustorff-Silva J, De Oliveira-Filho AG. Cross-sectional study comparing different therapeutic modalities for cystic lymphangiomas in children. Clinics. 2014; 69: 505-508.

10. Damaskos C, Garmpis N, Manousi M et al. Cystic hygroma of the neck: single center experience and literature review. Eu Re Med Pharmacol Sci. 2017; 21: 4918-4923.

11. Hesham Y, Hasan A, RIzwan MA. Efficacy of OK-432 therapy for the incisionless treatment of head and neck cystic masses. Sultan Qaboos University Med J. 2018; 18: 88-92. 